\title{
Non-pharmacological Interventions a Feasible Option for Addressing Dementia-related Sleep Problems in the Context of Family Care
}

Rosemary Gibson ( $\sim$ r.gibson@massey.ac.nz )

Massey University - Wellington Campus https://orcid.org/0000-0002-3747-095X

Anthony Dowell

University of Otago Wellington

Linda Jones

Massey University - Wellington Campus

Philippa Gander

Massey University - Wellington Campus

\section{Research}

Keywords: Actigraphy, Carers, Dementia, Non-pharmacological intervention, Sleep

Posted Date: September 15th, 2020

DOl: https://doi.org/10.21203/rs.3.rs-58103/v1

License: (c) (1) This work is licensed under a Creative Commons Attribution 4.0 International License.

Read Full License

Version of Record: A version of this preprint was published at Pilot and Feasibility Studies on May 26th, 2021. See the published version at https://doi.org/10.1186/s40814-021-00851-x. 


\section{Abstract}

Background: Sleep disturbances are challenging symptoms associated with mild cognitive impairment or dementia (MCloD). This study assessed the feasibility of sleep monitoring and non-pharmacological interventions to improve the sleep of New Zealanders with MCloD and their family carers.

Methods: A five-week multi-modal intervention consisting of timed bright light therapy, physical activity, and sleep education was piloted. Sleep was monitored for a week at baseline and conclusion of the trial using actigraphy, diaries, and questionnaires alongside additional health and wellbeing information concerning both care recipients and carers.

Results: Fifteen pairs participated, 9 completed the trial. Patterns of attrition and participant feedback are discussed. Case studies showed that six of the care recipients had minor improvements to sleep efficiency. Some also had improved subjective sleep ratings and quality of life. Changes did not clearly translate to family carers. However, five of them also showed some improvements in sleep status and mental health. Health deterioration of care recipients may mask the effects of the intervention.

Conclusions: It is feasible to use non-pharmacological sleep interventions for people with MCloD and their family carers. Given the limited treatment options, further consideration of such interventions in future research and clinical practice is warranted.

Trial registration: As this study was to assess the feasibility of proposed methods, it was an observational study without case-control groups and therefore did not require registration. A future full version of the trial would be registered with the Australian New Zealand Clinical Trails Registry.

\section{Key Messages Regarding Feasibility}

- Research was required in order to ascertain the feasibility of collecting sleep data and trialling nonpharmacological interventions among community dwelling, as opposed to institutionalised, people with dementia alongside their family carers

- It is feasible to collect subjective and objective sleep data from this population. While all methods were considered acceptable, sleep diaries were challenging due to the fragmented nature of sleep among care recipients. Non-pharmacological interventions were piloted successfully. However, the variability between subjects and rapid changes associated with dementia challenge the viability of a randomised controlled trail.

- These interventions have potential for improving the sleep and wellbeing of older people at risk or diagnosed with dementia. A person-centred approach incorporating mixed methods is recommended in order to maintain an ethical and feasible larger scaled study.

\section{Background}


Disordered, irregular sleep, and sleep deprivation have been associated with poorer cognition, physical and mental health, and mortality. 1,2 With aging, sleep typically deteriorates due to multiple physiological and psychological factors. ${ }^{3,4}$ As life expectancy rises, cases of mild cognitive impairment and dementia are increasing. ${ }^{5}$ Sleep disturbances often occur in the early stages of cognitive decline and have been identified as among the most challenging symptoms associated with progressive dementias (such as Alzheimer's Disease) ${ }^{6,7}$ These disturbances have been related to reduced activity and function of the circadian master clock in the hypothalamus, as well as psycho-social and behavioural factors. ${ }^{6}$ Not surprisingly, the sleep of family carers is also often affected. ${ }^{8}$ Situations where a person with mild cognitive impairment or dementia (MCloD) and their family carer are both sleeping poorly tend to be those which are more difficult to manage. ${ }^{8,9}$

Pharmacological options for the symptoms of MCloD (including sleep disturbances) often have side effects and limited effectiveness. ${ }^{10}$ There is great emphasis on exploring non-pharmacological strategies to modify and manage the symptoms within the individual's home environment. ${ }^{5}$ Better management of sleep health could lead to improvements in waking symptoms and wellbeing for people with MCloD as well as family carers. Interventions using timed bright light and/or physical activity can help stimulate the circadian system whilst promoting overall sleep health. ${ }^{11,12}$ Previous studies have found that interventions combining bright light therapy with physical or social activities as well as sleep hygiene education can promote more robust sleep timing and less disturbed sleep amongst people with dementia. 11-14 However, community-based trials are lacking and the feasibility of conducting such research in New Zealand (NZ) has yet to be explored. This is necessary because the NZ context differs from previous studies with regards to population size, cultural diversity, as well as sleep and age-related healthcare services.

\section{Aims and Objectives:}

1. To pilot a community-based approach for gathering objective and subjective data on the sleep of people with MCloD and their family carer in NZ. This included evaluating the acceptability and usability of the protocol and measures and identifying the most appropriate outcome variables.

2. To design and examine the feasibility of a trial for testing non-pharmacological interventions to improve sleep and waking function. This included measuring the impact of the intervention and identifying factors affecting participant compliance and withdrawal.

\section{Methods}

\section{Participants}


Fifteen pairs including a person with MCloD ("care recipients") and their family carers ("carers") were recruited via Alzheimers Wellington (a local organisation offering support and advocacy services for families affected by dementia) as well as via community advertisements, presentations, and referrals from healthcare professionals. Due to the pilot nature of the study, convenience sampling was used. Inclusion criteria were living together in the Wellington community, the care recipient being aged $\geq 65$ years, and that they and/or their carer had an interest in improving their sleep. Due to the underdiagnoses

of dementia, ${ }^{5}$ status of MCloD was based on participants' report and involvement with dementia-related services. Exclusion criteria were having eye conditions or taking medications which could cause photosensitivity to the light therapy element of the protocol. ${ }^{15}$

Written consent was gathered from each pair. Verbal assent was also recorded during the study briefing, to offer protection and/or an alternative for those with reduced cognitive capacity. Participant's doctors were informed and given the opportunity to notify the researcher of any further contraindications for bright light exposure or contact the research team with any concerns.

\section{Materials}

Sleep/wake activity of the pairs was measured for 7 days at baseline (Time 1) and during the fifth week of the intervention (Time 2) using the Actiwatch-2 (Mini Mitter, Philips, Respironics) worn on the nondominant wrist. The Actiwatch-2 has a button serving as an event marker for participants to press at relevant times (e.g. at bedtime), and a sensor for measuring the amount and duration of ambient white light exposure in units of lux (from the wrist). Activity counts were recorded in 1-minute intervals and the records were analysed using custom software provided by the manufacturer, using the medium sensitivity threshold. Time in bed was defined via the event marker and light data from the Actiwatch-2 and a sleep diary in the form of 24-hour timelines. Participants recorded the times of sleep start and end for any sleep that was 10 minutes or longer. Diaries for the care recipients were maintained throughout the intervention to collect information on compliance.

Questionnaires were provided to the pairs at Times 1 and 2. These included demographic information as well as validated questionnaires concerning sleep ${ }^{15,16}$, health ${ }^{17,18}$, symptoms and quality of life of those with $\mathrm{CloD}^{19-21}$ and the impact of care ${ }^{22}$ (see Table 1). Open-ended questions also prompted feedback on the protocol and ratings of each aspect of the methods and intervention. This information was evaluated regardless of whether a pair completed the entire protocol. 
Table 1

Content of questionnaires completed by care recipients and carers

\section{Completed by:}

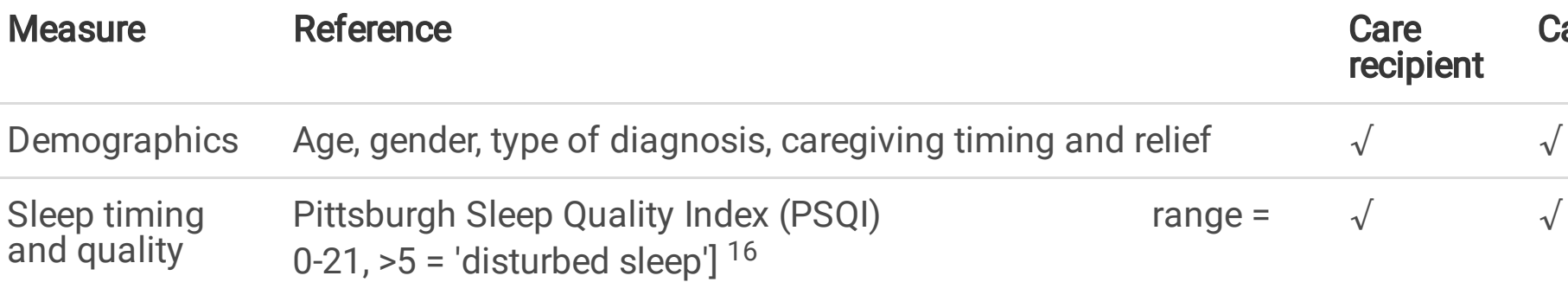

Dementia- Sleep Disorders Inventory (SDI)

related sleep $\quad 12 /$ 'severely disturbed sleep' 17

range $=0-$

disturbance

Health

Self-Administered Comorbidity Questionnaire (SCQ ${ }^{18)}$ and medication list

Cognitive Mini-Mental State Examination (MMSE) range $=30-0,>23=$ 'no $\sqrt{ }$

impairment impairment', 19-23 = 'mild cognitive impairment', 10-18 = 'moderate cognitive impairment', and $\leq 9$ = 'severe cognitive impairment' 19

Dementia- $\quad$ Revised Memory and Behaviour Problems Checklist (RMBPC) related global score (0-96) plus sub-scores for memory (range $=0-28$,

symptoms depression (range $=0-36$ ), and disruptive behaviours (range 0

- 32) with higher scores indicating increased frequency of

behaviours and negative carer affect ${ }^{20}$

Quality of life Quality of Life in Alzheimer's Disease: Patient and Caregiver

of recipient

Report (QOL-AD) range $=52$ (excellent) -13 (poor) ${ }^{21}$

Mental health Hospital Anxiety and Depression Scale (HAD), range 0-21, 0-7 = 'normal', 8-10 = 'borderline', 11-21 = heightened risk 22

Impact of

Carers of Older People in Europe index (COPE) range for

caregiving positive impact of caring items $=15-0(<12=$ reduced coping).

Range for negative impact of caring items $=0-18(>12=$

reduced coping) ${ }^{23}$

\section{Procedure}

Figure 1 provides an overview of study procedures. Questionnaires were completed by the care recipient in the presence of the researcher and carer, to support completion. In cases when they were not cognitively able, the carer acted as a proxy. The interventions included timed bright light therapy, exercise, and sleep hygiene education. A Day-Light light box was provided (Uplift Technologies Inc., 2009) as an 
alternative to natural light. This provides up to 10,000 lux of illumination at a comfortable sitting distance (30-33 centimetres). ${ }^{23}$ The proposed exercise regime was 30-40 minutes of walking or yoga style stretches and movement (participants were loaned a senior's exercise DVD and were given information about safe home exercise). A 'Sleep Support Handbook' was developed for the study that contained information on sleep with ageing and dementia, and sleep hygiene guidelines.

During the 5-week trial, care recipients were encouraged to get 30 minutes of bright light exposure every morning (or on as many mornings as possible) between 9-11am, using either the light box or natural light. They were also asked to undertake the low intensity exercises on as many days as possible during the middle of the day (11am-2pm). Carers were encouraged to join in with physical activities or supervise as they saw necessary. Finally, participants were encouraged to try some of the ideas/solutions offered in the handbook, and to note in the sleep diaries instances when they had tried new things. Participants were telephoned by the researcher on a weekly basis to remind them of the study protocol and answer any questions regarding the study. They also had access to a free phone number to contact the researcher throughout for advice or assistance.

\section{Data Analysis}

Actigraphy records were analysed with the diary data to identify rest periods. Fifty percent of records were double scored and anomalous responses and outliers were checked (average agreement rate $=84.8 \%$ for the care recipient records, $92.1 \%$ for carers). Sections of complete inactivity (due to the actiwatch not being worn or for unknown reasons) were excluded. Rest periods were categorised into night and day. Night was defined as the time the participant initially went to bed intending to sleep (bedtime) until the final morning rising (rise time). Standard actigraphy variables were derived from the software (e.g. total sleep time, sleep efficiency, number of awakenings) and compared for each participant between Times 1 and 2. Global scores were calculated for each validated questionnaire and its subscales. For scales with established threshold values, participants were categorised according to whether they scored above or below the thresholds. The Pittsburgh Sleep Quality Index (PSQI) was key, with scores above 5 indicating problematic sleep. ${ }^{15}$

Compliance with the intervention was estimated from the diaries (calculated from the number of days with light or activity logged within and outside of the requested time frames) as well as by comparing Time 1 light (9am-11am) and activity data (11am-2pm) from the Actiwatch 2's to Time 2 data. Descriptive statistics from the nominal questions in the feedback form were calculated and field notes from home visits and telephone conversations collated. Due to the small and heterogeneous sample, the effect and feasibility of the protocol and intervention was based on case studies. ${ }^{24}$ Individual scores were plotted for within-subject comparisons between Times 1 and 2 , and to visually compare the distribution of results between participants who completed the trial versus those who did not.

\section{Results}


Fifteen pairs took part. The demographic details of the care recipients and their study outcome are in Table 2. All but two pairs were spouses (pairs 6 and 14 were parent and child). The majority $(n=11)$ of the carers were not in paid employment. However, four were also working 20-40 hours per week (ID's 5, 6, 8 and 14). Summary data for key questionnaire and actigraphic data are provided in Table 3 . This shows that care recipients had poorer and more variable objective sleep duration and efficiency compared to carers. In contrast, care recipients' PSQI sores generally indicated less subjective sleep disturbance than carers. 
Table 2

Brief Demographic Details with Outcome Summaries
ID Age Gender
Diagnosis
MMSE
Carer Age
Outcome summary

\section{Those who} completed:

\begin{tabular}{ccccccc}
1 & 73 & Male & VD & 15 & 73 & $\begin{array}{l}\text { Dementia-related deterioration, minor } \\
\text { improvements to Care recipient's PSQI } \\
\text { and actigraphic sleep }\end{array}$ \\
\hline 2 & 82 & Male & VD & 13 & 74 & $\begin{array}{l}\text { Dementia-related deterioration, PSQI } \\
\text { improved for both }\end{array}$ \\
\hline 4 & 72 & Male & AD & 14 & 71 & $\begin{array}{l}\text { Good quality sleep at onset, minor } \\
\text { improvement to care recipient's PSQI }\end{array}$ \\
\hline 46 & Male & Undefined & 23 & 74 & $\begin{array}{l}\text { Good quality sleep at onset, no } \\
\text { improvements observed, care recipient } \\
\text { had marked daytime sleepiness }\end{array}$
\end{tabular}

$\begin{array}{llllll}5 & 82 & \text { Male } & \text { LBD } & 5 & 68 \quad \begin{array}{l}\text { Marked improvements to both subjective } \\ \text { and actigraphic sleep variables as well as } \\ \text { waking function of care recipient }\end{array}\end{array}$

$6 \quad 83 \quad$ Female Undefined $27 \quad 46 \quad$ Minor subjective and actigraphic improvements to sleep, however carer reported deterioration to care recipient's sleep

\begin{tabular}{|c|c|c|c|c|c|c|}
\hline 7 & 73 & Female & $A D$ & 16 & 78 & $\begin{array}{l}\text { Good quality sleep at onset, no } \\
\text { improvements observed }\end{array}$ \\
\hline 8 & 66 & Male & $A D$ & 18 & 67 & $\begin{array}{l}\text { Good sleep quality at onset, no } \\
\text { improvements observed. Struggled with } \\
\text { protocol }\end{array}$ \\
\hline
\end{tabular}

$9 \quad 80 \quad$ Male $\quad$ VD $\quad 7 \quad 54 \quad$ Minor improvements to actigraphic sleep of both. Carer reported stress and sleep disturbed by care recipient

Those who withdrew:

\begin{tabular}{|ccccccl}
10 & 80 & Female & AD & 21 & 81 & $\begin{array}{l}\text { Withdrew due to care recipient moving } \\
\text { into care home at end of week 1 }\end{array}$ \\
\hline 11 & 72 & Male & AD & 13 & 68 & $\begin{array}{l}\text { Withdrew as care recipient admitted to } \\
\text { hospital }\end{array}$ \\
\hline 12 & 88 & Male & MCl & 29 & 75 & $\begin{array}{l}\text { Withdrew after losing motivation after } \\
\text { period of respite care }\end{array}$ \\
\hline 13 & 83 & Female & VD & 20 & 82 & $\begin{array}{l}\text { Withdrew at the end of week 1, sleep not } \\
\text { considered problematic enough }\end{array}$ \\
\hline 14 & 89 & Female & Undefined & 23 & 55 & $\begin{array}{l}\text { Withdrew as intervention protocol } \\
\text { considered too stressful }\end{array}$
\end{tabular}




\begin{tabular}{|lllllll}
\hline ID & Age & Gender & Diagnosis & MMSE & $\begin{array}{l}\text { Carer } \\
\text { Age }\end{array}$ & Outcome summary \\
\hline 15 & 73 & Male & VD & 28 & 55 & $\begin{array}{l}\text { Withdrew during week } 1 \text { due to care } \\
\text { recipient moving into care facility after a } \\
\text { fall }\end{array}$ \\
\hline $\begin{array}{l}\text { Abbreviations: VD = Vascular dementia, AD = Alzheimer's disease, LBD = Lew Body Dementia MCl } \\
=\end{array}$ \\
$\begin{array}{l}\text { Mild Cognitive Impairment, } \\
\text { Endefined = type of dementia unknown/not specified in diagnosis, MMSE = Mini Mental Status }\end{array}$ \\
\hline
\end{tabular}


Table 3

Descriptive statistics and paired summaries of key variables for participants who started and completed the protocol (first and second line for each variable respectively).

\begin{tabular}{|c|c|c|c|c|c|c|}
\hline \multirow{2}{*}{ Variable (range of scale) } & \multicolumn{3}{|c|}{ Time 1} & \multicolumn{3}{|c|}{ Time 2} \\
\hline & $\mathbf{N}$ & Median & (range) & $\mathbf{N}$ & Median & (range) \\
\hline \multicolumn{7}{|l|}{ Care recipient } \\
\hline \multirow[t]{2}{*}{ Sleep duration night, hours } & 15 & 8.0 & $(5.5-10.6)$ & & & \\
\hline & 9 & 8.1 & $(6.7-9.8)$ & 9 & 8.2 & $(5.7-12.3)$ \\
\hline \multirow[t]{2}{*}{ Sleep efficiency night (\%) } & 15 & 85.0 & $(61.3-98.4)$ & & & \\
\hline & 9 & 85.0 & $(62.0-95.9)$ & 9 & 85.4 & $(61.4-96.5)$ \\
\hline \multirow[t]{2}{*}{ Sleep duration day, minutes } & 15 & 47.0 & $(0.0-78.0)$ & & & \\
\hline & 9 & 51.5 & $(0.0-78.0)$ & 9 & 29.5 & $(0.0-192.0)$ \\
\hline \multirow[t]{2}{*}{ PSQI (0-21) } & 15 & 4.0 & $(1.0-13.0)$ & & & \\
\hline & 9 & 4.0 & $(1.0-8.0)$ & 9 & 3.0 & $(2.0-7.0)$ \\
\hline \multirow[t]{2}{*}{ SDI-AD $(0-12)$} & 14 & 0.2 & $(0.0-4.2)$ & & & \\
\hline & 9 & 0.9 & $(0.0-4.0)$ & 9 & 1.1 & $(0.0-3.0)$ \\
\hline \multirow[t]{2}{*}{ MMSE $(30-0)$} & 14 & 18.0 & $(7.0-29.0)$ & & & \\
\hline & 9 & 15.0 & $(5.0-27.0)$ & 9 & 15.0 & $(6.0-26.0)$ \\
\hline \multirow[t]{2}{*}{ RMBPC Behaviour frequency $(0-32)$} & 15 & 3.5 & $(0.0-11.0)$ & & & \\
\hline & 9 & 3.0 & $(0.0-11.0)$ & & 5.0 & $(2.0-10.0)$ \\
\hline \multirow[t]{2}{*}{ RMBPC Behaviour reaction $(0-32)$} & 15 & 3.5 & $(0.0-19.8)$ & & & \\
\hline & 9 & 3.3 & $(0.0-12.0)$ & 9 & 3.5 & $(0.0-24.0)$ \\
\hline \multirow[t]{2}{*}{ QOL-AD (CR rated), $(52-13)$} & 14 & 39.0 & $(24.0-48.0)$ & & & \\
\hline & 8 & 39.5 & $(24.0-45.0)$ & 9 & 36.0 & $(18.0-49.0)$ \\
\hline \multirow[t]{2}{*}{ QOL-AD (C rated), $(52-13)$} & 15 & 30.0 & $(23.0-40.0)$ & & & \\
\hline & 9 & 30.0 & $(23.0-40.0)$ & 9 & 34.0 & $(22.0-40.0)$ \\
\hline \multicolumn{7}{|l|}{ Carer } \\
\hline \multirow[t]{2}{*}{ Sleep duration night, hours } & 14 & 7.5 & $(5.9-8.9)$ & & & \\
\hline & 8 & 7.9 & $(6.1-8.9)$ & 7 & 7.6 & $6.7-9.1$ \\
\hline
\end{tabular}




\begin{tabular}{|c|c|c|c|c|c|c|}
\hline \multirow[b]{2}{*}{ Sleep efficiency (\% sleep night) } & \multicolumn{3}{|c|}{ Time 1} & \multicolumn{3}{|c|}{ Time 2} \\
\hline & 14 & 89.6 & $(72.5-93.7)$ & & & \\
\hline & 8 & 90.0 & $(85.1-93.7)$ & 7 & 87.1 & $(83.3-92.4)$ \\
\hline \multirow[t]{2}{*}{ Sleep duration day, minutes } & 14 & 7.0 & $(0.0-80.0)$ & & & \\
\hline & 8 & 0.0 & $(0.0-80.0)$ & 7 & 0.0 & $(0.0-47.5)$ \\
\hline \multirow[t]{2}{*}{ PSQI (0-21) } & 14 & 8.0 & $(1.0-10.0)$ & & & \\
\hline & 8 & 8.0 & $(1.0-9.0)$ & 9 & 5.0 & $(2.0-9.0)$ \\
\hline \multirow[t]{2}{*}{ COPE positive $(15-0)$} & 15 & 11.0 & $(4.0-14.0)$ & 9 & 10.0 & $(4.0-15.0)$ \\
\hline & 9 & 10.0 & $(4.0-14.0)$ & & & \\
\hline \multirow[t]{2}{*}{ COPE negative $(0-18)$} & 15 & 5.0 & $(1.0-12.0)$ & 9 & 10.0 & $(0.0-12.0)$ \\
\hline & 9 & 6.0 & $(1.0-12.0)$ & & & \\
\hline \multirow[t]{2}{*}{ HADS-Anxiety $(0-21)$} & 15 & 4.0 & $(0.0-12.0)$ & 9 & 6.0 & $(1.0-12.0)$ \\
\hline & 9 & 4.0 & $(0.0-12.0)$ & & & \\
\hline HADS-Depression (0-21) & 15 & 4.0 & $(0.0-9.0)$ & 9 & 5.0 & $(0.0-11.0)$ \\
\hline \multicolumn{7}{|c|}{$\begin{array}{l}\text { PSQI = Pittsburgh Sleep Quality Index; SDI-AD = Sleep Disorders Inventory Alzheimer's Disease; MMSE } \\
=\text { Mini Mental Status Exam; RMBPC = Revised Memory Behaviour Problem Checklist (global scores } \\
\text { unavailable due to missing data); QOL-AD = Quality of Life in Alzheimer's disease" Patient and } \\
\text { Caregiver report; COPE = Carers of Older People in Europe index; HADS = Hospital Anxiety and } \\
\text { Depression Scale }\end{array}$} \\
\hline
\end{tabular}

Six of the pairs withdrew during the trial. Care recipients in these pairs tended to be less cognitively impaired (median MMSE $=20.5$, range $=13.0-29.0$ ) compared to those who completed the trial (median MMSE $=15.5$, range $=7.0-27.0)$. However, the RMBPC scores indicated that those who withdrew tended to have more frequent and disruptive behaviours (median $=6.3$, range $=0.0-10.5 \mathrm{vs}$. median $=3.0$, range $=0$ 11). The carers reaction rating to these behaviours was also higher amongst those who withdrew ( median $=5.8$, range $=0-19.8$ ) compared to those who completed the trial (median $=3.0$, range $=0-12$ ). Two withdrawal "types" were identified amongst the care recipients. Firstly, those who had less severe symptoms of dementia with minor sleep problems and lost interest or were confused by the study protocol (Pairs 12, 13, and 14); and secondly, those who had more advanced symptoms of dementia with substantial sleep problems but were admitted to hospital or a care facility during the trial period and so could not complete the trial (Pairs 10,11, and 15). All pairs rated the actigraphy and questionnaires as okeasy to use. Only one rated the diary as difficult, but some carers noted the challenge of being able to reliably document sleep and wake bouts across the 24-hr day for care recipients who had very fragmented sleep. For those who completed the trial ( $n=9$ pairs), diary-reported compliance with the bright light therapy ranged from $37 \%-97 \%$ of the days during the five-week intervention (median $72 \%$ ).

Compliance with the exercises was lower (3\%-86\% days, median 55\%). The interventions were not always 
used in the specified timeframes (56\% of the time and $38 \%$ of the time respectively). The Actiwatch 2 data showed an $85 \%$ increase in mean light exposure for care recipients within the 9am-11am timeframe between baseline and trial completion (mean $\mathrm{T} 1=4,905$ lux, $\mathrm{SD}=5,377$ lux vs. mean $\mathrm{T} 2=9,077, \mathrm{SD}=$ $14,709)$ compared to just a $2 \%$ increase in physical activity counts within the $11 \mathrm{am}-2 \mathrm{pm}$ timeframe (mean activity counts $T 1=27,635, S D=15,096$ vs. mean activity counts $T 2=28,181, S D=13,338$ ). There was no clear pattern of baseline measures being reliable predictors for trial outcomes. Feedback data revealed that none of the pairs found the light box difficult to use. However, two found the exercise DVD difficult and one reported difficulty with the education booklet. Seven of the nine said that they would recommend the interventions to others (the others said they maybe would).

Six of the care recipients showed minor improvements in their sleep efficiency at night (0.6-3.6\%, See Figure 2a). Their median time in bed was 10.2 hours (range $=8.7-11.9$ hours), indicating an increase of 4-22 minutes more sleep at Time 2 . While the grouped data are variable, there were cases with more marked improvements in sleep and wellbeing (e.g. Participant 5, case study presented elsewhere). ${ }^{24}$ The majority (six) of the care recipients scored normal/good on the PSQI at Time $1(<5)$. Nevertheless, five showed 1-3 points of improvement on their PSQI scores at Time 2 (see Figure 3a). Actigraphic and subjective improvements in sleep (PSQI or SDI scores) were not clearly related. Of the five care recipients who had improved PSQI ratings at Time 2, two also had improved scores on the MMSE (3-4 points) and three had improvements to their quality of life as rated by their carer QOL-AD (2-7 points). However, between Times 1 and 2, six had reductions of 1-7 points in their MMSE score. Furthermore, many of the behavioural symptoms associated with dementia (as indicated by participant feedback and scores on the RMBPC or QOL-AD) deteriorated across the trial period.

Six out of the nine carers had PSQI scores that indicated disturbed sleep $(>5)$ at Time 1. Five had 1-5 points of improvement in their PSQI scores between Times 1 and 2 (see Figure 3a). Of these five, three also had decreased depression scores at Time 2 (as indicated by the HADS). However, the majority (seven) scored within the normal range for symptoms of anxiety and depression at Time 1. Improvements to the care recipient's sleep did not always translate into improved sleep, mood or coping of their family carer (Figures 2 and 3).

\section{Discussion}

This study indicates that a combined intervention using bright light therapy and/or therapeutic exercise with sleep hygiene education is feasible for people with $\mathrm{MCloD}$ and their family carers at home. Given the paucity of options for treating dementia, the intervention has potential for improving the sleep and wellbeing of older people at risk or diagnosed with dementia. The mixed-methods approach and use of case studies including both care recipients and care partners was novel, allowing investigation into the context of conducting such a study in the NZ community. The protocol was considered feasible and suggestions for future trials were obtained. The small heterogeneous sample limited the ability to measure the clinical effectiveness of the interventions, or to determine the characteristics of those most likely to benefit. However, some pairs did report benefits to their sleep and waking wellbeing. In other 
cases, a rapid deterioration of health masked any possible improvements or prevented participants from being able to complete the trial. These mixed findings are concordant with previous research with people living in institutions or the community, using both cross-sectional studies and randomised controlled trials ${ }^{25}$. They are representative of the clinical reality of families affected by dementia.

The interventions offer a low-risk, drug-free option for managing dementia-related sleep disturbances. They were designed to address well-defined physiological and psychosocial processes affecting sleep and waking function (most notably stabilising the function of the circadian master clock). ${ }^{6,7}$ The protocol in the present study differed from previous community-based trials e.g.13, 26 in that bright light was recommended at mid-morning, using a higher intensity and for a shorter duration. The exercise component also had a recommended time frame. Delivering the interventions this way was considered more suitable for the participants, since dementia-related symptoms and behaviours often exacerbate towards evening, which is also a time of high care demands. ${ }^{6}$ The timing of the light and exercise exposures was also anticipated to minimise the likelihood of the interventions having an arousing effect on the care recipient before bedtime. ${ }^{11,12}$

Compared to previous community-based studies, ${ }^{13,26}$ the present sample of care recipients had more varied types of dementia-related impairment. They also had less problematic sleep at baseline, as indicated by both actigraphic and subjective reports as well as higher baseline light exposure (more than 2,000 lux). Similarly, the sample of carers had relatively good actigraphic sleep compared to those in previous samples. However, their subjective ratings indicated a high prevalence of sleep problems which was more comparable. ${ }^{8}$ Discrepancies between subjective and objective sleep data of carers is not unusual and likely associated with the psychosocial impact of caring on the symptoms of insomnia. ${ }^{27}$ Carers in the present sample appeared to have reduced likelihood of anxiety or depression compared to previous samples of NZ carers ${ }^{23}$, which may have contributed to different sleep outcomes.

Variable improvement in sleep problems associated with MCloD may be attributable to a range of factors. These include variability in comorbid diseases and demographic factors, the fluctuating nature of sleep and dementia-related behaviours, as well as response bias, possible placebo effects, and the impact of researcher support. Comparisons between studies are also confounded by differences in sleep measures and intervention characteristics. The course of cognitive deterioration and factors necessary for stable sleep timing and efficiency (including damage to the eye, pineal gland, and circadian system as well as presence of another primary sleep disorder) are also expected to affect intervention effectiveness. ${ }^{25}$

Based on the present case studies, it is recommended that future trials are tailored towards care recipients who have limited access to bright light and physical activity, as well those with poor sleep status at baseline. A larger randomised controlled trial would enable greater monitoring of the impact of the intervention while controlling for biases. The Inclusion of qualitative elements in such trials is recommended to strengthen our understanding of the psychosocial elements of sleep health with ageing, 
$\mathrm{MCl}$ and dementia as well as providing a person-centred perspective on the effectiveness of the interventions in this highly variable population.

Considering the variation between families affected by dementia as well as issues with recruitment, compliance, and attrition in the present study, future trials may benefit from a more personally tailored approach to engagement and delivery as well as a reduced research load on the participants. This could be achieved through automated rather than diary-assisted scoring of actigraphy data (removing the need for keeping dairies), ${ }^{28}$ customising the interventions, and delivering them via primary healthcare or preexisting home care services with a support person to facilitate compliance with the intervention(s). Such an approach has been used with other health promotion interventions for older people ${ }^{\text {e.g. } 29}$ and would likely facilitate recruitment and retention of participants, as well enabling better evaluation of the impact of the intervention and its broader use in aged-care services.

\section{Conclusion}

With a rapidly ageing population, increases in New Zealanders affected by dementia are projected. Many are supported at home by a family carer and or wider family/ whānau. This model is becoming increasingly critical for regulating the costs to the healthcare system as well as for facilitating living well with advancing age and dementia ${ }^{5,30}$. This study indicates that a combined non-pharmaceutical intervention for sleep is feasible for people with MCloD and their family carers at home, with limited research burden. While the effect of the intervention was variable at the group level, some individuals reported improvements to sleep and waking status. We conclude that, given the lack of options for treating dementia, the intervention has potential for improving the sleep and wellbeing of older people at risk or diagnosed with dementia and further trials are warranted.

\section{List Of Abbreviations}

MCloD, mild cognitive impairment or dementia.

\section{Declarations}

\section{Ethics approval and consent to participate}

Approval was obtained from the Central Health and Disability Ethics Committee (CEN/11/01). Written consent to participate was gathered from each pair by the lead researcher (RG). Verbal assent was also recorded during the study briefing, to offer protection and/or an alternative for those with reduced cognitive capacity.

\section{Consent for publication}


Consent for reporting and publication of anonymised data was included in initial information sheets and consent process.

\section{Availability of data and materials}

The protocol, study documents, and datasets produced and analysed during the current study are available from the corresponding author on reasonable request. In alignment with the Health and Disability Ethics Committee of NZ regulations, data are stored for 10 years in electronic and paper form in secure locations at the Sleep/Wake Research Centre and will be unavailable after July 2022.

\section{Competing interests}

The authors declare that they have no competing interests

\section{Funding}

Funders of this research include the Health Research Council of New Zealand feasibility grant (number 11/562), and Maurice and Phyllis Paykel Trust (project and equipment grant, 2010). Neither funding body had any role with regards to the design of the study or the collection, analysis, or interpretation of data nor in writing the manuscript.

\section{Authors' contributions}

RG designed the study protocol and applied for funding, recruited participants and collected data. RG also led the analyses and write up of the results. LJ contributed to the study with regards to engagement of people with dementia living in the community and interpretation of results. TD informed the study with regards to its application with clinical practice and community healthcare. PG oversaw the study, informing the study design and protocol with regards to their expertise in circadian health, applying for funding, guiding interpretation of results. All authors read and approved the final manuscript."

\section{Acknowledgements}

We would like to acknowledge the participants of this study and Kanchana Pathirana for assistance with data entry.

\section{References}

1. Adams RJ, Appleton SL, Taylor AW, et al. Sleep health of Australian adults in 2016: results of the 2016 Sleep Health Foundation national survey. Sleep Health. 2017;3. 
2. Da Silva AA, De Mello RGB, Schaan CW, et al. Sleep duration and mortality in the elderly: A Systematic review with meta-analysis. BMJ Open. 2016;6:e008119. doi:10.1136/bmjopen-2015008119.

3. Ohayon MM, Carskadon MA, Guilleminault C, Vitiello MV. Meta-analysis of quantitative sleep parameters from childhood to old age in healthy individuals: Developing normative sleep values across the human lifespan. Sleep. 2004;27.

4. Scullin MK, Bliwise DL. Sleep, Cognition, and Normal Aging: Integrating a Half Century of Multidisciplinary Research. Perspectives on Psychological Science. 2015;10.

5. Alzheimers New Zealand Incorporated. Dementia economic impact report 2016. 2017: Available from: http://www.alzheimers.org.nz/reports-and-statistics/.

6. Vitiello MV, Borson S. Sleep disturbances in patients with Alzheimer's disease: Epidemiology, pathophysiology and treatment. Drugs. 2001;15.

7. Saeed Y, Abbott SM. Circadian Disruption Associated with Alzheimer's Disease. Curr Neurol Neurosci Rep. 2017;17: doi:10.1007/s11910-017-0745-y.

8. McCurry SM, Logsdon RG, Teri L, Vitiello MV. Sleep disturbances in caregivers of persons with dementia: Contributing factors and treatment implications. Sleep Med Rev. 2007;11.

9. Gibson RH, Gander PH, Jones LM. Understanding the sleep problems of people with dementia and their family caregivers. Dementia. 2014;13.

10. McCleery J, Cohen DA, Sharpley AL. Pharmacotherapies for sleep disturbances in dementia. Cochrane Database Syst Rev. 2016;11:CD009178.

11. Buxton OM, Lee CW, L'Hermite-Balériaux $M$, et al. Exercise elicits phase shifts and acute alterations of melatonin that vary with circadian phase. Am J Physiol Regul Integr Comp Physiol. 2003;284.

12. Khalsa SBS, Jewett ME, Cajochen C, Czeisler CA. A phase response curve to single bright light pulses in human subjects. J Physiol. 2003;549.

13. McCurry SM, Pike KC, Vitiello MV, et al. Increasing walking and bright light exposure to improve sleep in community-dwelling persons with Alzheimer's disease: Results of a randomized, controlled trial. J Am Geriatr Soc. 2011;59.

14. Martin JL, Marler MR, Harker JO, et al. A multicomponent nonpharmacological intervention improves activity rhythms among nursing home residents with disrupted sleep/wake patterns. J Gerontol A Biol Sci Med Sci. 2007;62.

15. Buysse DJ, Reynolds ICF, Monk TH, et al. The Pittsburgh Sleep Quality Index: A new instrument for psychiatric practice and research. Psychiatry res. 1989;28.

16. Tractenberg RE, Singer CM, Cummings JL, Thal LJ. The Sleep Disorders Inventory: An instrument for studies of sleep disturbance in persons with Alzheimer's disease. J Sleep Res. 2003;12.

17. Sangha O, Stucki G, Liang MH, et al. The Self-Administered Comorbidity Questionnaire: A new method to assess comorbidity for clinical and health services research. Arthritis Rheum. 2003;49.

18. Zigmond AS, Snaith RP. The Hospital Anxiety and Depression Scale. Acta Psychiatra Scand. 1983;67. 
19. Folstein MF, Folstein SE, McHugh PR. 'Mini mental state'. A practical method for grading the cognitive state of patients for the clinician. J Psychiatr Res. 1975;12.

20. Teri L, Truax P, Logsdon R, et al. Assessment of behavioral-problems in dementia - The revised memory and behavior problems checklist. Psychol Aging. 1992; 7.

21. Logsdon RG, Gibbons LE, McCurry SM, Teri L. Assessing quality of life in older adults with cognitive impairment. Psychosom Med. 2002;64.

22. Roud $H$, Keeling $S$, Sainsbury R Using the COPE assessment tool with informal carers of people with dementia in New Zealand. NZ Med J. 2006; 119 (1237): U2053.

23. Wirz-Justice A, Benedetti F, Terman M. Chronotherapies for affective disorders: A clincian's manual for light and wake therapy. Basel: Karger; 2008.

24. Gibson RH, Gander PH, Jones LM, Dowell AC. Non-pharmacological interventions for managing dementia-related sleep problems within community dwelling pairs: A mixed-method approach. Dementia. 2016;16.

25. Brown CA, Berry R, Tan MC, et al. A critique of the evidence base for non-pharmacological sleep interventions for persons with dementia. Dementia. 2013;12.

26. Friedman L, Spira AP, Hernandez B, et al. Brief morning light treatment for sleep/wake disturbances in older memory-impaired individuals and their caregivers. Sleep Med. 2012;13.

27. Rowe MA, McCrae CS, Campbell JM, et al. Sleep pattern differences between older adult dementia caregivers and older adult noncaregivers using objective and subjective measures. J Clin Sleep Med. 2008;4.

28. Gibson RH, Gander PH. Monitoring the sleep patterns of people with dementia and their family carers in the community. Australas J Ageing. 2019; 38.

29. Parsons $\mathrm{M}$, Senior $\mathrm{H}$, Kerse $\mathrm{N}$, et al. Randomised trial of restorative home care for frail older people in New Zealand. Nurs Older People. 2017;29.

30. Dyall L. Dementia. Continuation of health and ethnic inequalities in New Zealand. NZ Med J. 2014;127.

\section{Figures}




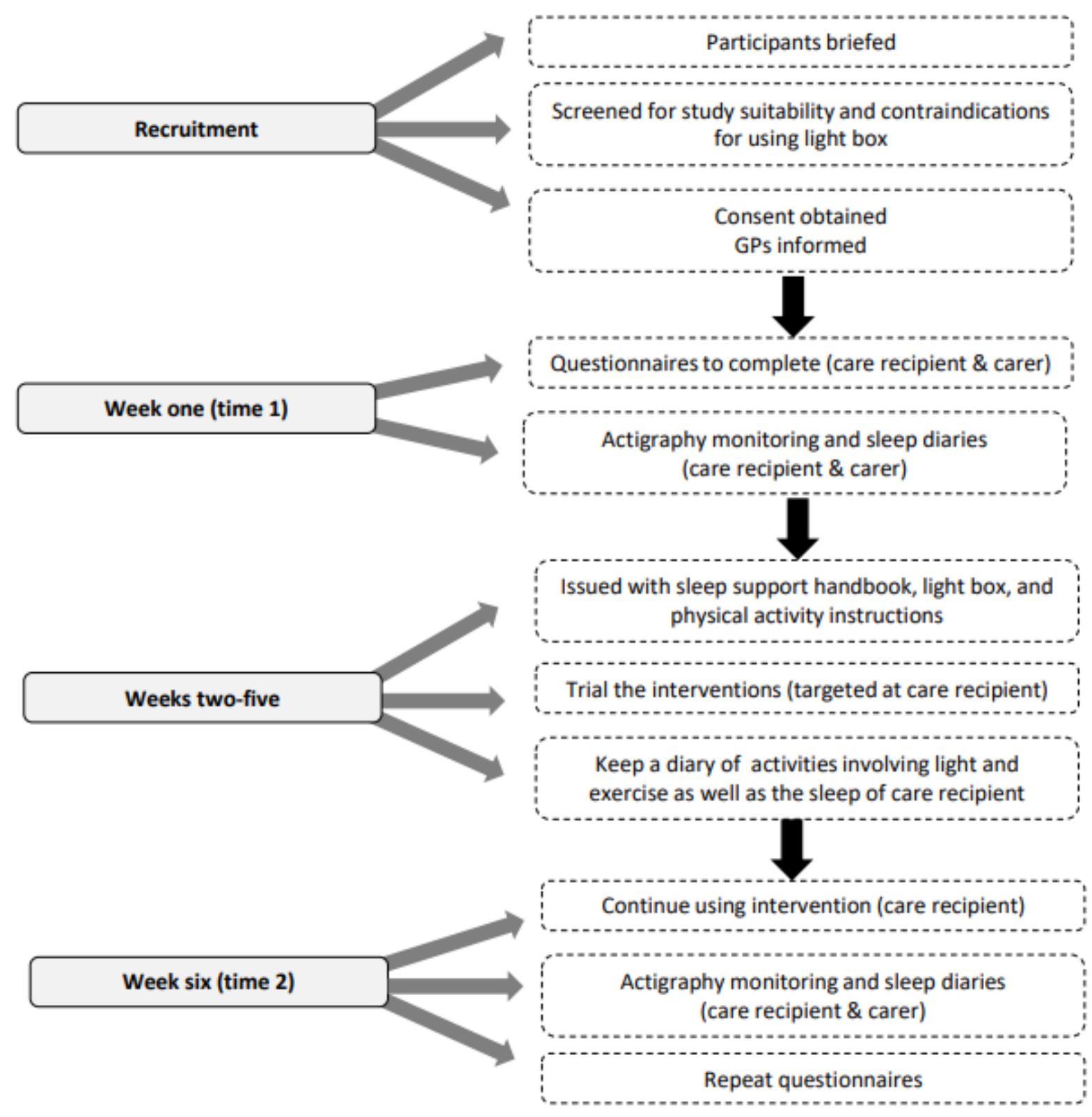

\section{Figure 1}

Overview of the study protocol 

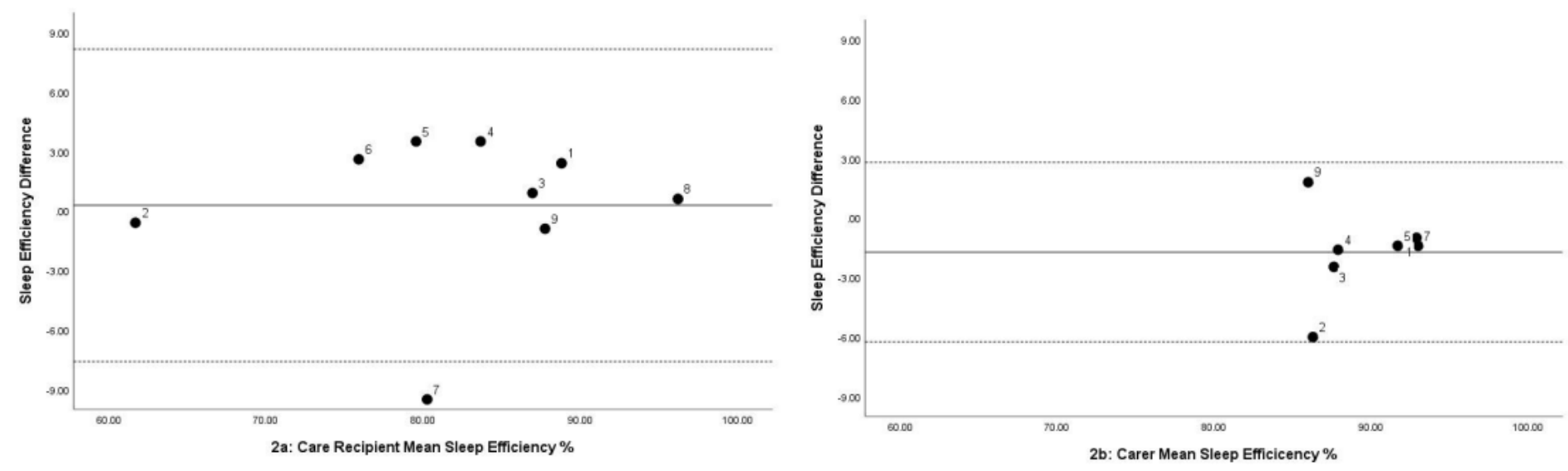

Figure 2

Bland Altman plots comparing sleep efficiency at night between times 1 and 2 for care recipients (2a) and family carers $(2 b)$
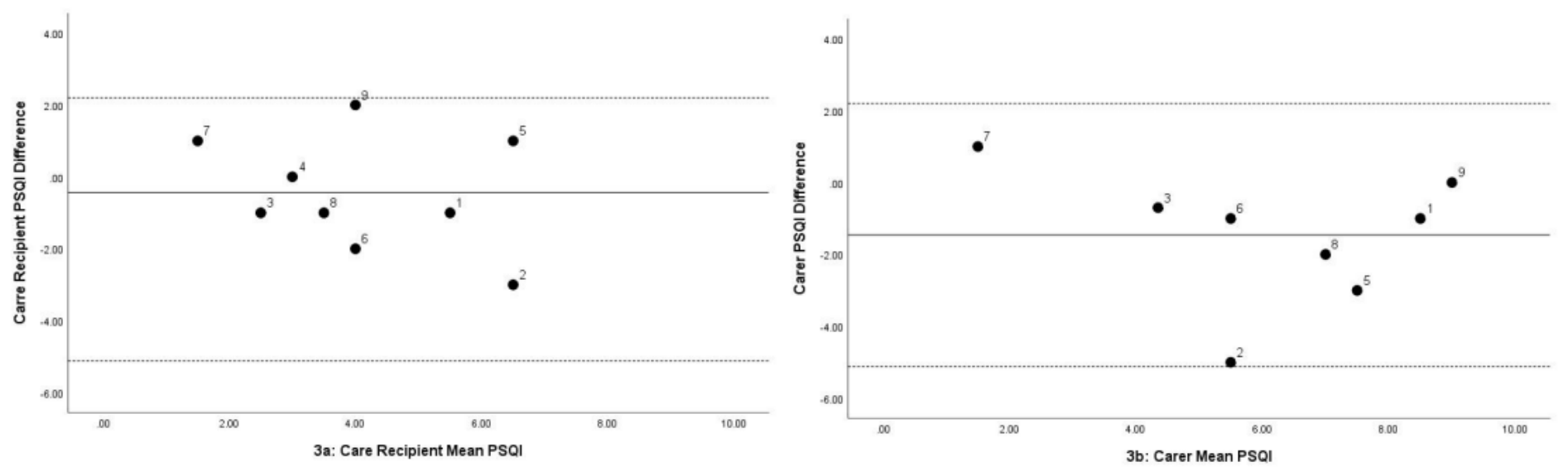

Figure 3

Bland Altman plots comparing PSQI scores between times 1 and 2 for care recipients ( $3 a$ ) and family carers (3b)

\section{Supplementary Files}

This is a list of supplementary files associated with this preprint. Click to download.

- SPIRITChecklistdownload8Jan13RGpilot.doc 